\title{
Bulk Photoemission from Plasmonic Nanoantennas of Different Shapes
}

\author{
Renat Sh. Ikhsanov, ${ }^{*}+\odot$ Andrey V. Novitsky, ${ }^{\ddagger}$, Igor E. Protsenko," and Alexander V. Uskov ${ }^{\| \odot}$ \\ ${ }^{\dagger}$ National Research University Higher School of Economics, 101000 Moscow, Russia \\ ${ }^{\ddagger}$ Technical University of Denmark, 2800 Kongens Lyngby, Denmark \\ ${ }^{\S}$ Belarusian State University, 220030 Minsk, Belarus \\ "P. N. Lebedev Physical Institute, Leninsky pr. 53, 119991 Moscow, Russia
}

ABSTRACT: We elaborate a semi-analytical model for calculation of the bulk internal emission of photoelectrons from metal nanoparticles into a semiconductor matrix. We introduce important effects in the model as the jump of the effective electron mass at the metal-semiconductor interface and cooling of the hot electrons because of electron-electron and electron-nanoparticle surface collisions in the metal. We study the interplay between the plasmonic electric dipole and quadrupole resonances and reveal the optimum parameters for different geometrical shapes of nanoparticles with respect to
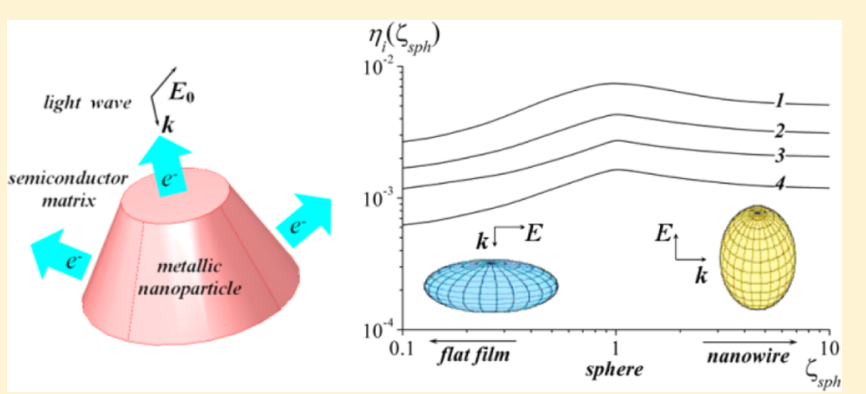
the photoemission cross section. We find that the absorption cross section well-predicts the optimum size of the dipolar nanoparticle. This opens the possibility for the fast optimization and design of the photoelectric devices.

\section{INTRODUCTION}

Generation of hot electrons in plasmonic nanoantennas, that is, in nanometer-sized metal particles, with subsequent emission of the hot electrons into the surrounding semiconductor matrix attracts growing interest because of its potential applications in photochemistry and photocatalysis, light harvesting (solar cells and nano-photodetectors), and optoelectronics. ${ }^{1-5}$

Resonant electron photoemission from nanoantennas occurs under the excitation of "localized surface plasmon resonances" (LSPR) of collective oscillations of the electron density in metal nanoantennas by external quasi-monochromatic electromagnetic field with a carrier frequency equal to LSPR frequency. With the excitation of LSPR, a significant increase of the electromagnetic field inside nanoantennas and, as a consequence, enhanced absorption of electromagnetic energy by electrons of metal occur. If the energy of hot electrons, generated upon absorption, is sufficient for overcoming of the potential barrier at the matrix-nanoantenna interface, then the LSPR excitation is accompanied by a resonant increase in photoemission. Because LSPR depends strongly on shape and size of nanoparticles, the resonant photoemission also depends on these characteristics of nanoantennas. Note that LSPR in large nanoparticles can be broadened because of the radiative losses, ${ }^{6}$ whereas in small nanoantennas the resonance is broadened because of electron collisions with nanoparticle boundary. ${ }^{7,8}$

It is well-known that the surface and volume photoelectric effects can occur in plasmonic nanoantennas. ${ }^{3,9}$ Surface photoelectric effect is characterized by absorption of photons by electrons at their collision with the interface between the nanoparticle and the surrounding medium. If electrons, during collision, receive enough energy from photons to overcome the potential barrier at the interface, they are emitted from the metal. In bulk photoeffect, electrons absorb quanta of electromagnetic field oscillations inside the nanoparticle at their collisions with phonons or defects of the metal crystal lattice. Then, the hot electrons move to the boundary and either overcome the barrier at the boundary or reflect back into the volume of the nanoparticle (see Figure 2). On the way to the boundary, the hot electron cools down at collisions with cold electrons of the metal, phonons, and so on. The cooling of the hot electrons is characterized by the mean free path length, ${ }^{10}$ which, as well as LSPR, depends on the nanoantenna size and shape. Tamm and Schubin in ref 11 indicated that the surface photoemission can be stronger than the volume one. On the basis of this result, we developed the theory of electron photoemission from plasmonic nanoantennas for the surface mechanism first (see ref 9). Later (in ref 3), we compared surface and volume photoeffects from metallic nanospheres into the surrounding semiconductor and found the regime, when the volume photoeffect dominates. This explains our motivation and interest to the volume mechanism of photoemission from nanoantennas in addition to surface photoemission.

One should note that very recently it was demonstrated experimentally ${ }^{12,13}$ that surface photoeffect (named "coherent

Received: February 27, 2018

Revised: $\quad$ May 4, 2018

Published: May 10, 2018 
photoemission" in ref 12) can be enhanced substantially by special resonant states at the metal-semiconductor interface. These results are very important for further development of the surface photoeffect and nanoantennas as sources of hot electrons, but their consideration is outside of the scope of present paper.

In this paper, we study the bulk photoemission. In particular, we calculate (1) internal quantum efficiency (IQE) of the photoemission and (2) photoemission cross section (PCS). One should note that in ref 4, we restricted ourselves with calculations of only IQE for nanostructures of the simplest shapes (flat thin film, nanowire, and nanosphere) and did not study plasmonic resonance properties of nanostructures. Here, we also consider PCS that characterizes "external quantum efficiency" and properly reveals plasmonic nanoantenna properties. In section 2, we present theoretical background of bulk photoemission for plasmonic nanoparticles of arbitrary shape following the approach of $\operatorname{refs}^{3,4,9,10}$ and introduce formulas for the IQE and PCS of a nanoparticle. In section 3, we present computational tools (software) used in our calculations and display the set of parameters employed in our model. Section 4 describes calculations of IQE and PCS for examples of nanoparticles in a homogeneous semiconductor medium: for spheroidal particles with various aspect ratios and for truncated cones with various conicity parameters and sizes. The results and the outlook for further research are discussed in Conclusions.

\section{THEORY}

2.1. Basic Characteristics of the Photoemission. We consider a single metal nanoparticle with the complex permittivity $\varepsilon_{\mathrm{i}}(\omega)=\varepsilon_{\mathrm{i}}^{\prime}(\omega)+\mathrm{i} \varepsilon_{\mathrm{i}}^{\prime \prime}(\omega)$ of the metal, embedded into a semiconductor matrix with real-valued permittivity, $\varepsilon_{\mathrm{e}}$ (see Figure 1). The nanoparticle is illuminated by a plane electromagnetic wave of the angular frequency $\omega$ and amplitude $E_{0}$. The illumination induces the field of the amplitude $E_{\mathrm{i}}(r)$ inside the nanoparticle, and this field is partially absorbed by electrons of the metal. The absorption is characterized by the absorption cross section ${ }^{3,6}$

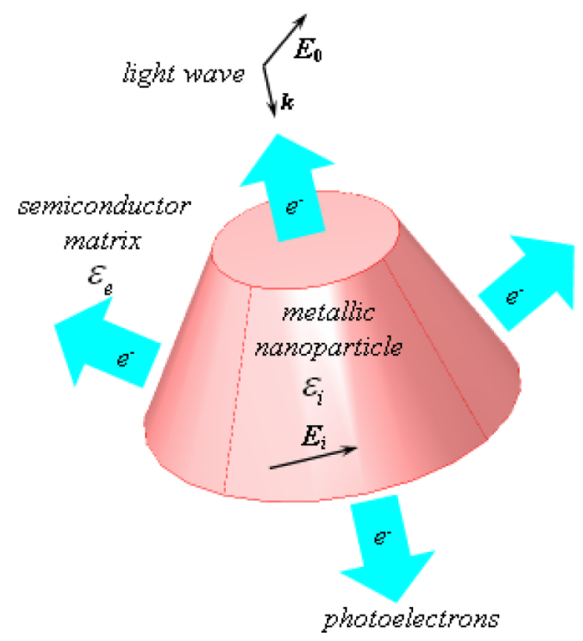

Figure 1. Metal nanoparticle in a semiconductor matrix illuminated by resonant light. The incident light, plane wave with an electric field amplitude $E_{0}$ and wave vector $k$, induces electric field $\boldsymbol{E}_{\mathrm{i}}$ inside the nanoparticle. $\varepsilon_{\mathrm{i}}$ and $\varepsilon_{\mathrm{e}}$ are the dielectric permittivities of metal and semiconductor, respectively.

$$
\sigma_{\mathrm{abs}}(\lambda)=\frac{2 \pi}{\lambda} \frac{\varepsilon_{\mathrm{i}}^{\prime \prime}}{n_{\mathrm{e}}}\left|\boldsymbol{E}_{0}\right|^{-2} \int_{V_{\mathrm{n}}} \boldsymbol{E}_{\mathrm{i}}^{2}(\boldsymbol{r}) \mathrm{d}^{3} r
$$

where $c$ is the speed of light in vacuum, $\lambda=2 \pi c / \omega$, and $n_{\mathrm{e}}=\sqrt{\varepsilon_{\mathrm{e}}}$ is the refractive index of the matrix. Integration in eq 1 is performed over the nanoparticle volume $V_{\mathrm{n}}$.

Absorption of the electromagnetic energy leads to generation of hot electrons inside the nanoparticle, and such hot electrons (photoelectrons) can be emitted from the nanoparticle. The electron photoemission is characterized by the PCS $\sigma_{\text {phe }}$ defined as a ratio of the emission rate of photoelectrons from the nanoparticle to the flux of photons of the external field incident on the nanoparticle. Introducing the "local internal quantum efficiency of photoemission (LIQE)" $\eta_{\mathrm{i}}^{\text {local }}(\boldsymbol{r})$ as the probability of generation of hot electrons at the point $r$ of the nanoparticle, $\sigma_{\text {phe }}$ can be written as ${ }^{3,4}$

$$
\sigma_{\text {phe }}(\lambda)=\frac{2 \pi}{\lambda} \frac{\varepsilon_{\mathrm{i}}^{\prime \prime}}{n_{\mathrm{e}}}\left|\boldsymbol{E}_{0}\right|^{-2} \int_{V_{\mathrm{n}}} \eta_{\mathrm{i}}^{\text {local }}(\boldsymbol{r}) \boldsymbol{E}_{\mathrm{i}}^{2}(\boldsymbol{r}) \mathrm{d}^{3} r
$$

Then, the quantum efficiency of photoemission from the nanoparticle reads

$$
\eta_{\mathrm{i}}=\sigma_{\mathrm{phe}}(\lambda) / \sigma_{\mathrm{abs}}(\lambda)
$$

Below, we calculate the LIQE $\eta_{\mathrm{i}}^{\text {local }}(\boldsymbol{r})$ and the PCS $\sigma_{\text {phe }}(\lambda)$.

2.2. Model of Photoemission. We follow the approach of ref 10 and take into account the scattering of photoelectrons on imperfections in the nanoparticle. The model of ref 10 allows us to obtain analytical expressions for IQE and is based on the four assumptions (see Figures 2 and 3).

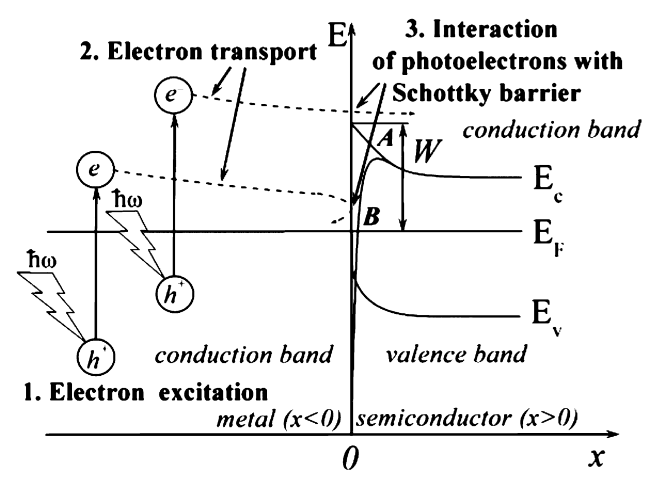

Figure 2. Scheme of the bulk photoemission process (band diagram): 1 -photoexcitation of hot electrons of the metal at the collisions with phonons or with lattice defects (the transition is from the occupied energy states in the conduction band to the unoccupied states above the Fermi level $E_{\mathrm{F}}$ ); 2-hot electron transport in the metal; 3interaction of hot electrons with a Schottky barrier (passing through the barrier or reflection from it). $E_{\mathrm{v}}$ and $E_{\mathrm{c}}$ are the energies of the top of the valence band and of the bottom of the conduction band of the semiconductor far from the interface, respectively. Curves $E_{\mathrm{c}}(x)$ marked by $A$ and $B$ show the Schottky barrier taken without $(A)$ and with $(B)$ the image forces. $W$ is the work function at the metalsemiconductor interface.

1) Electron-phonon scattering is elastic. In fact, electron energy loss in a single collision with a phonon is about $10^{-3} \mathrm{eV}$, while the mean free path of electrons in electron-phonon collisions is estimated as $50 \mathrm{~nm}$ for gold and silver. ${ }^{14}$ This means that the losses of hot photoelectrons are no more than tenths of a percent of the initial hot electron energy on the way from the point 


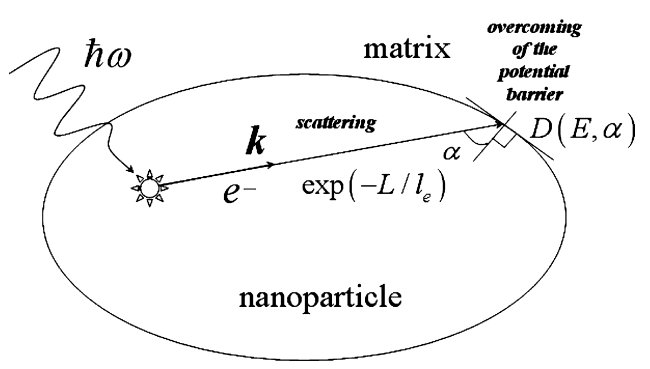

Figure 3. Illustration of propagation of a hot electron in a nanoparticle.

of the hot electron generation to the nanoparticle boundary. Such losses can be neglected for nanoparticles with typical dimensions (as a diameter for a sphere) less than $100 \mathrm{~nm}$.

2) In the first event of hot-“cold” electron scattering, a photoelectron loses approximately half of its excess (relative to the Fermi level) energy. ${ }^{14}$ After that the hot electron is not able to overcome the potential barrier at the metal-semiconductor interface. Therefore, the only hot electron having no collisions with "cold" electrons on its way to the boundary can be emitted. Thus, we can treat a photoelectron's path to the boundary as a segment of a straight line. In this case, the probability of reaching the boundary without electron-electron collisions is $\exp \left(-L / l_{\mathrm{e}}\right)$, where $L$ is the distance from the point of generation of the hot electron to the point of its collision with the boundary and $l_{\mathrm{e}}$ is the mean free path of electron-electron scattering.

3) Strictly speaking, the length $l_{\mathrm{e}}$ depends on hot electron's energy $\Delta E_{\mathrm{f}}$ over the Fermi level, that is, $l_{\mathrm{e}}=l_{\mathrm{e}}\left(\Delta E_{\mathrm{f}}\right)$ (see refs 15 and 16). However, this dependence can be neglected in calculations, when a relatively narrow range of photon energies near the long-wavelength limit is considered. For instance, the mean free path $l_{\mathrm{e}}$ for gold changes from $\sim 40$ to $\sim 42 \mathrm{~nm}$ for the range of hot electron energies $1 \mathrm{eV} \leq \Delta E_{\mathrm{f}} \leq 2 \mathrm{eV}$ (see ref 16). Therefore, we take the constant value $l_{\mathrm{e}}=41 \mathrm{~nm}$ in our calculations.

4) Multiple reflections of the hot electrons from the boundary are neglected.

With the assumptions (1-4), the probability for a photoelectron created at the point $r$ of the nanoparticle to cross the interface reads

$$
P_{\mathrm{em}}(\boldsymbol{r}, \boldsymbol{k})=D\left(E(k), \alpha\left(\boldsymbol{r}, \boldsymbol{e}_{\mathrm{k}}\right)\right) \exp \left(-L\left(\boldsymbol{r}, \boldsymbol{e}_{\mathrm{k}}\right) / l_{\mathrm{e}}\right)
$$

where $k$ is electron's wave vector, $D$ is the transmission coefficient for the potential barrier at the interface, $\alpha$ is the incident angle of the photoelectron, and $e_{\mathrm{k}}=k /|k|$ is the unit vector along $k$.

Then, if we neglect the thermal excitation of electrons, we can write LIQE as

$$
\eta_{\mathrm{i}}^{\text {local }}(\boldsymbol{r})=\frac{\int_{V_{\mathrm{k}}} P_{\mathrm{em}}(\boldsymbol{r}, \boldsymbol{k}) \rho(k) \mathrm{d}^{3} k}{\int_{V_{\mathrm{k}}} \rho(k) \mathrm{d}^{3} k}
$$

where $\rho(k)$ is the density of electron energy states in $k$-space.

We assume that the dispersion relation for electrons in metal is parabolic $\left(E \propto k^{2}\right)$ and $\rho(k) \propto 1 / 4 \pi^{3}$, where $k$ belongs to the spherical layer $k_{\mathrm{F}}<k<k_{\hbar \omega}$ of the volume

$$
V_{\mathrm{k}}=4 \pi\left(k_{\hbar \omega}^{3}-k_{\mathrm{F}}^{3}\right) / 3
$$

with $\hbar k_{\mathrm{F}}=\sqrt{2 m E_{\mathrm{F}}}$ and $\hbar k_{\hbar \omega}=\sqrt{2 m\left(E_{\mathrm{F}}+\hbar \omega\right)}$.

The cooling of hot electrons can be modeled quite precisely within the Monte Carlo method ${ }^{14}$ but it is very computationally demanding. Because of that, several approximate methods have been developed. ${ }^{3,10,17,18}$ Our model taken from ref 10 is fast and simple. This model has been already employed for numerical and analytical calculations in ref 4.

Now, we discuss the model for calculation of the transmission coefficient $D$ of the potential barrier. We exploit the approximation of local flatness of the metal-semiconductor interface. It is valid, when electron's de Broglie wavelength is much smaller than the radius of curvature of the nanoparticle surface at the point of electron's photoemission. We use the assumption of specular reflection of electrons from the boundary, commonly adopted in the theory of the photoelectric effect, ${ }^{19}$ when the wave vector of the reflected electron lies in the plane of the incidence and angles of reflection and incidence are equal.

There are various involved approximations for calculations of potential barriers on the metal-semiconductor boundary (e.g., image forces can be taken into account as in ref 20). Here, we use simple approximation that the barrier shape is a rectangular potential step. As a benefit for exploiting this approximation, one can easily find the exact quantum-mechanical expression for the probability of electron passage through the barrier, when the jump of the effective electron mass at the interface is taken into account.

Detailed calculation of the transmission coefficient of the potential barrier has been performed in refs 20 and 21 . Here, we approximate the barrier with a rectangular potential step of the height equal to the work function from the metal into the semiconductor ( $W$ in Figure 2 ). Then, we can use the wellknown quantum-mechanical expression for the transmission coefficient and take into account the jump of the effective electron mass at the interface. This jump is important, because the effective masses of electrons in the metal and semiconductor may differ more than in an order of magnitude. For example, $\mathrm{m} / \mathrm{m}^{*}$ is about 4 for $\mathrm{Au} / \mathrm{Si}$ and about 15 for the $\mathrm{Au}-$ GaAs interface, ${ }^{22}$ where $m$ and $m^{*}$ are the effective masses of electrons in the metal and semiconductor matrix, respectively. The jump in effective masses significantly affects the effective barrier height and consequently the quantum yield of the photoemission. $^{23,24}$

Thus, we take the transmission coefficient is as in ref 4

$$
D=\operatorname{Re}\left\{4 \frac{\left[r_{\mathrm{m}}\left(1-U_{\rho} / E_{\mathrm{z}}\right)\right]^{1 / 2}}{\left(1+\left[r_{\mathrm{m}}\left(1-U_{\rho} / E_{\mathrm{z}}\right)\right]^{1 / 2}\right)^{2}}\right\}
$$

where $E_{\rho}=E \sin ^{2} \alpha, E_{\mathrm{z}}=E \cos ^{2} \alpha, E=\hbar^{2} k^{2} / 2 m, r_{\mathrm{m}}=m / m^{*}$, and $U_{\rho}=W+\left(r_{\mathrm{m}}-1\right) E_{\rho}$.

Our model for the dielectric permittivity $\varepsilon_{\text {eff }}$ of metallic nanoparticles takes into account the broadening of plasmonic resonances because of electron collisions with the nanoparticle boundary $^{8}$

$$
\varepsilon_{\text {eff }}(\omega)=\varepsilon_{\mathrm{i}}(\omega)+\mathrm{i} \Delta \varepsilon_{\text {surf }}^{\prime \prime}(\omega)
$$

where $\varepsilon_{\mathrm{i}}$ is the bulk permittivity of the metal and $\Delta \varepsilon_{\text {surf }}^{\prime \prime}$ is a correction to the imaginary part of the bulk permittivity caused by collisions of electrons with the nanoparticle surface. $\Delta \varepsilon_{\text {surf }}^{\prime \prime}$ depends on the frequency and polarization of the incident light, 
as well as on the shape of the nanoparticle. We calculate $\Delta \varepsilon_{\text {surf }}^{\prime \prime}$ for the truncated cone nanoparticle (in particular, for the cylinder nanoparticle) following the procedure described in ref 8 for spheroidal nanoparticles. In ref 8 , the shape of a nanoparticle is characterized by the shape parameter

$$
F_{\text {shape }}=L_{c} S / V
$$

where $S, V$, and $L_{c}$ are the nanoparticle surface area, volume, and characteristic size, respectively. For a spheroidal nanoparticle, $L_{\mathrm{c}}$ is a length of the spheroid semiaxis perpendicular to its rotation axis $\left(L_{c}=b\right.$, see the inset in Figure 4$)$, then $F_{\text {shape, }}$ as

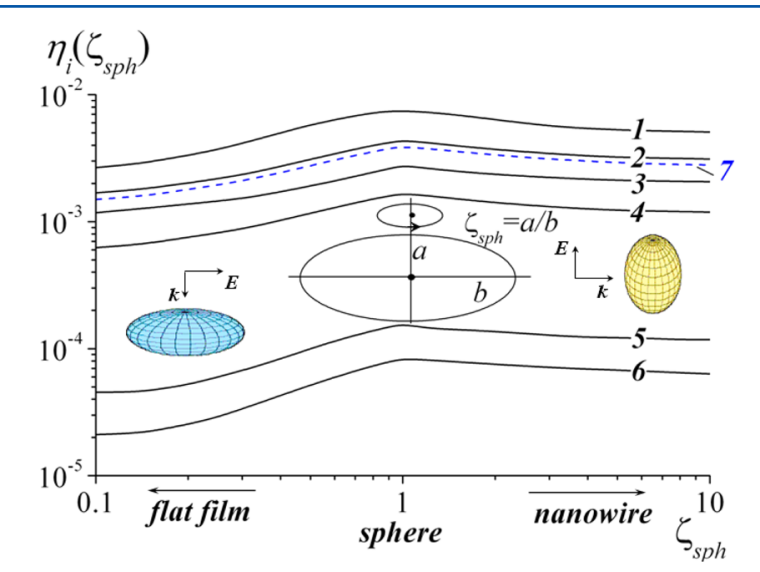

Figure 4. IQE for a spheroidal nanoparticle as a function of its aspect ratio $\zeta_{\text {sph. }}$. The nanoparticle at $\zeta_{\text {sph }}=0$ is a sphere of $50 \mathrm{~nm}$ diameter; $a$ $\rightarrow \infty$ and $b=25 \mathrm{~nm}$ at $\varsigma_{\mathrm{sph}} \rightarrow \infty ; a=25 \mathrm{~nm}$ and $b \rightarrow \infty$ at $\varsigma_{\mathrm{sph}} \rightarrow 0$. The vacuum wavelength of light, illuminating the nanostructure, is $1000 \mathrm{~nm}$. The wave vector and polarization of the incident electromagnetic wave are shown in insets. The curves 1-6 are calculated for the Au-GaAs pair for different values of $l_{\mathrm{e}}$ and $r_{\mathrm{m}}: 1-l_{\mathrm{e}}$ $=\infty, r_{\mathrm{m}}=1 ; 2-l_{\mathrm{e}}=41 \mathrm{~nm}, r_{\mathrm{m}}=1 ; 3-l_{\mathrm{e}}=20 \mathrm{~nm}, r_{\mathrm{m}}=1 ; 4-l_{\mathrm{e}}=41$ $\mathrm{nm}, r_{\mathrm{m}}=2 ; 5-l_{\mathrm{e}}=41 \mathrm{~nm}, r_{\mathrm{m}}=10 ; 6-l_{\mathrm{e}}=41 \mathrm{~nm}, r_{\mathrm{m}}=15($ case of $\mathrm{Au}-\mathrm{GaAs}$ barrier). Curve 7 is calculated for the $\mathrm{Au}-\mathrm{Si}$ pair.

well as $\Delta \varepsilon_{\text {surf }}^{\prime \prime}$ depends only on the spheroid aspect ratio $\zeta_{\text {sph }}=$ $a / b$, where $a$ is the length of the spheroid semiaxis parallel to its rotation axis. Here, eq 7 for $F_{\text {shape }}$ is used also for the truncated cone, with $L_{c}$ to be the radius of the larger cone base (or any base of the cylinder). Taking such $F_{\text {shape }} \equiv F_{\text {shape }}^{\text {cone }}$, we find the effective aspect ratio for the truncated cone nanoparticle.

To calculate $\Delta \varepsilon_{\text {surf }}^{\prime \prime}$ we use the equation

$$
F_{\text {shape }}^{\text {spheroid }}\left(\varsigma_{\text {sph }}\right)=F_{\text {shape }}^{\text {cone }}
$$

where $F_{\text {shape }}^{\text {spheroid }}\left(\varsigma_{\mathrm{sph}}\right)$ is determined by eq 32 in ref $8\left(1 / \varsigma_{\text {sph }}\right.$ is designated there as $\left.r_{\text {asp }}\right)$. Then, by inserting $\varsigma_{\text {sph }}$ into eq 28 in ref 8 , we find $\Delta \varepsilon_{\text {surf }}^{\prime \prime}\left(\varsigma_{\text {sph }}\right)$.

\section{COMPUTATIONAL DETAILS}

For numerical calculations, we use Mathcad, MATLAB, and MNPBEM $^{25}$ software packages. MNPBEM package, being a toolbox of MATLAB, allows us to calculate the electric field distribution inside the nanoparticles [quantity $E_{\mathrm{i}}^{2}$ in eqs 2 and $3]$ and then the absorption $\sigma_{\text {abs }}$ and photoemission $\sigma_{\text {phe }}$ cross sections. Spatial distribution of the electric field in the nanoparticle is exploited by the Mathcad software to perform numerical integration. Thus, characteristics of the nanoparticles can be computed: IQE, absorption cross section, and PCS. The full set of parameters employed in our model includes

- $E_{\mathrm{F}}$, the Fermi level of the metal;
- $W$, the work function of escape from the metal into the semiconductor;

- $l_{\mathrm{e}}$, the hot-electron cooling length;

- $r_{\mathrm{m}}=\mathrm{m} / \mathrm{m}^{*}$, the ratio of the effective masses of an electron in the metal $(m)$ and semiconductor matrix $\left(m^{*}\right)$;

- $n_{\mathrm{e}}$, the semiconductor matrix refractive index;

- $\varepsilon_{\mathrm{i}}(\lambda)$, the nanoparticle metal permittivity;

- $D$, the diameter of the sphere;

- $a$ and $b$, the semiaxes of spheroidal nanoparticle (we also use parameter $\zeta_{\mathrm{sph}}=a / b$, see below);

- $D_{\text {cyl }}$ and $H_{\text {cyl }}$ the diameter and height of the cylinder, respectively;

- $R_{1}, R_{2}$, and $H$, the geometrical parameters of the truncated cone: radii of smaller and bigger bases and height of the nanoparticle, respectively (also, we use parameter $\zeta=1-R_{1} / R_{2}$, see below);

- $\sigma_{\text {geom }}$ the geometric cross section of a nanoparticle: $\pi D^{2} /$ $4, \pi D_{\text {cyl }}^{2} / 4$, and $\pi R_{2}^{2}$ for the sphere, cylinder, and truncated cone, respectively.

The calculations are carried out for the gold nanoparticles embedded in the homogeneous GaAs matrix. Unless otherwise specified (in some cases, the parameters take different values to show the influence of particular factor), the model parameters are equal to ${ }^{3,4} E_{\mathrm{F}}=5.51 \mathrm{eV}, W=0.8 \mathrm{eV}, l_{\mathrm{e}}=41 \mathrm{~nm}, m=1, m^{*}$ $=0.067$, and $n_{\mathrm{e}}=3.6$. Geometric parameters are specified in the text below.

Electric field distribution is calculated using the numerical solution of the full Maxwell equations ("retarded" mode of MNPBEM). The permittivity of Au was taken from ref 26.

\section{DEPENDENCE OF THE PHOTOEMISSION ON THE SHAPE OF NANOPARTICLES}

4.1. Spheroidal Nanoparticle. Uniform distribution of the electric field inside a small spheroidal nanoparticle can be found in the quasistatic limit. ${ }^{8}$ As one can see from eqs 2 and 3 , the PCS in this case is ${ }^{4}$

$$
\sigma_{\text {phe }}=\sigma_{\text {abs }} \eta_{\mathrm{i}}
$$

The IQE $\eta_{\mathrm{i}}$ of the spheroid is a function of its aspect ratio $\zeta_{\text {sph }}$. Aspect ratio $\zeta_{\text {sph }}=1$ corresponds to a sphere (here sphere's diameter is $50 \mathrm{~nm})$, at $\varsigma_{\text {sph }} \rightarrow 0$ the spheroid is transformed to a flat film (of the thickness $50 \mathrm{~nm}$ ), whereas at $\varsigma_{\text {sph }} \rightarrow+\infty$ one obtains a nanowire (infinite cylinder) of diameter $50 \mathrm{~nm}$.

We numerically calculate the IQE in a wide range of the values of $\zeta_{\text {sph }}$. Correctness of numerical calculations is confirmed by their correspondence to the analytical results at $\zeta_{\text {sph }} \rightarrow 0, \zeta_{\text {sph }}=1$, and $\zeta_{\text {sph }} \rightarrow+\infty$. As it is shown in Figure 4, the IQEs for a sphere, cylinder, and two-sided flat film with the same characteristic dimensions relate to each other approximately as $3: 2: 1$. This ratio slightly (with $20 \%$ deviations) depends on parameters of the model.

As an illustration of the role of electron cooling, we depict the curves 1, 2, and 3 in Figure 4 for various hot-electron cooling lengths $l_{\mathrm{e}}=\infty, 41$, and $20 \mathrm{~nm}$, respectively. Scattering processes characterized by the realistic $l_{\mathrm{e}}=41 \mathrm{~nm}$ reduces the IQE by a factor of about 1.5 . Curves $2,4,5$, and 6 demonstrate the dependence of $\eta_{\mathrm{i}}$ on the difference between the electron masses in the metal and semiconductor. The ratios of the masses for curves 2, 4, 5, and 6 are $r_{\mathrm{m}}=1, r_{\mathrm{m}}=2, r_{\mathrm{m}}=10$, and $r_{\mathrm{m}}=15$, respectively. According to Figure 4 the IQE decreases 

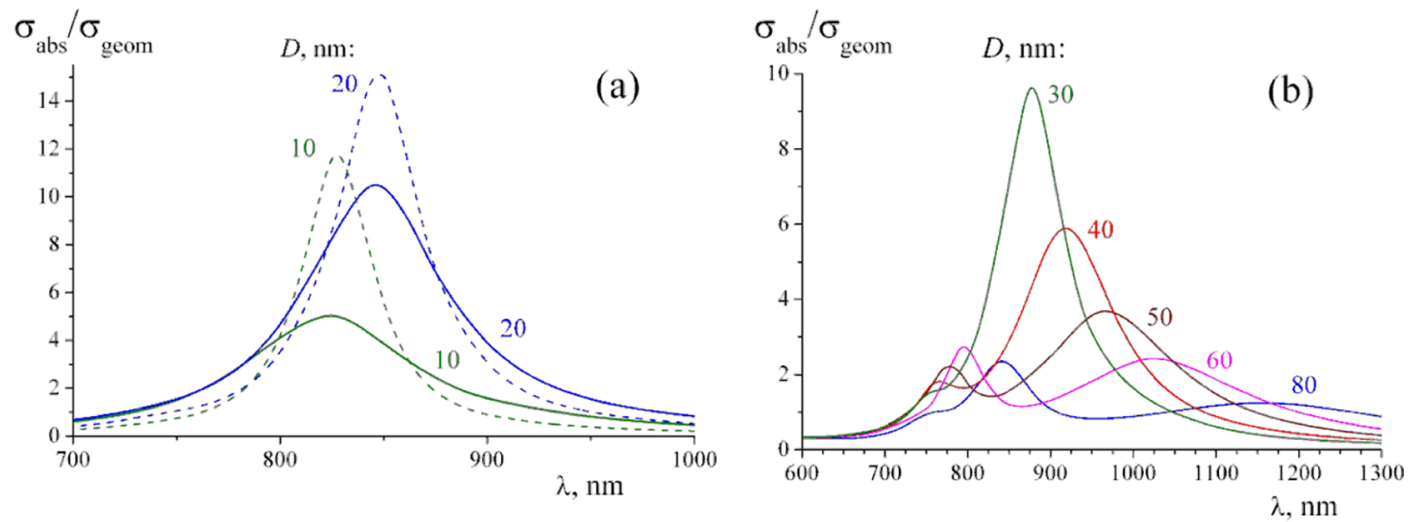

Figure 5. Absorption cross-section spectrum of the sphere $\sigma_{\text {abs }}$ normalized to its geometric cross section $\sigma_{\text {geom }}$ for various diameters $D$ of the sphere indicated near curves in nm. (a) Small nanoparticles. Plasmonic peaks with (solid curves) and without (dashed) electron collisions with the sphere boundary. (b) Large nanoparticles. One can notice the electric dipole (long-wavelength) and quadrupole (short-wavelength) resonances. Here and below, the graphs show the results for the gold nanoparticles and GaAs matrix.

by a factor of about 100 , when $r_{\mathrm{m}}$ increases from 1 to 15 . Thus, it is utterly necessary to take into account the jump of the effective electron mass at the metal-semiconductor interface in calculations of the IQE of metallic nanoparticles.

Despite the fact that we have chosen GaAs as the matrix, we cannot ignore silicon as the most popular material for inorganic photovoltaics. The work function of electron's escape from $\mathrm{Au}$ into p-type Si equals $W=0.34 \mathrm{eV}$; the ratio of the effective masses of an electron in the metal and semiconductor matrices $r_{\mathrm{m}} \approx 4$ (see ref 9). As it can be seen from Figure 4, the IQE of the sphere for $\mathrm{Au}-\mathrm{Si}$ system approximately (within 15\%) corresponds to the IQE of the $\mathrm{Au}-\mathrm{GaAs}$ system, if the jump of the effective electron mass in $\mathrm{Au}-\mathrm{GaAs}$ is not taken into account (compare the curves 2 and 7 ). If the jump is taken into account, then the $\mathrm{Au}-\mathrm{Si}$ system turns out to be about 50 times (in average) more effective than the $\mathrm{Au}-\mathrm{GaAs}$ one (compare the curves 6 and 7).

Our calculations confirm that the spheroid optimal for photoemission is a sphere. The question about the optimum diameter of the sphere at fixed material parameters for the nanoparticle and matrix is more complicated. Below, we optimize the photoemission and absorption cross sections normalized to the geometric nanoparticle cross section considering step by step all stages of the photoemission. The first stage is the absorption of a photon. Obviously, the optimized photoemission must be at maximum of the normalized absorption cross section.

Figures $5 \mathrm{a}, \mathrm{b}$ shows the evolution of the frequency dependence of the normalized absorption cross section with increase of sphere's diameter. Results for rather small dipolar spherical nanoparticles are presented in Figure 5a. Dashed curves in Figure 5a are calculated without taking into account correction $\Delta \varepsilon_{\text {surf }}^{\prime \prime}$ [see eq 6] because of collisions of electrons with the nanoparticle boundary. Collisions decrease the height of the plasmonic peak and broaden it, this effect being stronger for smaller diameters of the sphere. For larger spherical nanoparticles, one can observe both electric dipole and quadrupole resonant peaks as demonstrated in Figure 5b. Quadrupole resonance exists on the left to the dipole resonance and prevails over the dipole resonance for diameters of the nanoparticle greater than approximately $60 \mathrm{~nm}$.

Figure 6 shows correlation between the absorption $\sigma_{\mathrm{abs}}$ and photoemission $\sigma_{\text {phe }}$ cross-section spectra. $\sigma_{\text {phe }}$ is predicted by $\sigma_{\mathrm{abs}}$ for the short wavelengths but not for the long ones. The

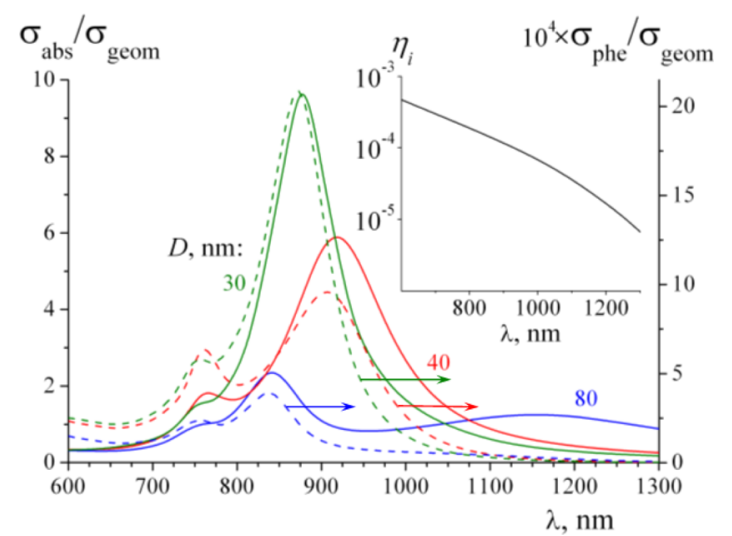

Figure 6. Absorption (solid curve) and photoemission (dashed) crosssection spectra for spheres of various diameters. Inset: IQE spectrum for a sphere of $40 \mathrm{~nm}$ diameter.

most significant deviation in the absorption and photoemission cross-section spectra occurs near the red boundary of the photoemission (1551 nm, in our case).

In Figure 6, one can notice that the dipole peak observed in the absorption cross-section spectrum for nanoparticles of diameter $80 \mathrm{~nm}$ disappears in the PCS spectrum. Such a discrepancy is explained by the strong wavelength dependence of the IQE $\eta_{i}$, the proportionality coefficient between $\sigma_{\text {phe }}$ and $\sigma_{\text {abs }}$ according to eq 8. $\eta_{\mathrm{i}}$ as a function of frequency is shown in the inset of Figure 6. $\eta_{\mathrm{i}}$ shifts the peaks of the absorption cross section to shorter wavelengths, and the shift is stronger close to the red boundary of the photoemission.

It should be noted that the well-known ${ }^{6}$ law of the absorption cross section as $\sigma_{\text {abs }} \propto D^{3}$ ceases to be satisfied because of the significant radiation losses for gold nanospheres embedded in the high-refractive index matrix $\left(n_{\mathrm{e}}=3.6\right.$ for GaAs). Curve 1 in Figure 7 is calculated in the quasistatic approximation [eq $5.13 \mathrm{~b}$ in ref 6] and shows a linear dependence of the normalized absorption cross section of the sphere on its diameter at the electric dipole resonance peak. Operation of taking the maximum over the wavelength in our case does not significantly affects the dependence $\propto D^{3}$, so the value $\max _{\lambda}\left(\sigma_{\mathrm{abs}}(\lambda) / \sigma_{\text {geom }}\right)$ increases linearly with $D$. However, taking into account the correction for radiation drastically changes the situation (compare curve 1 with curves 2 and 3 in 


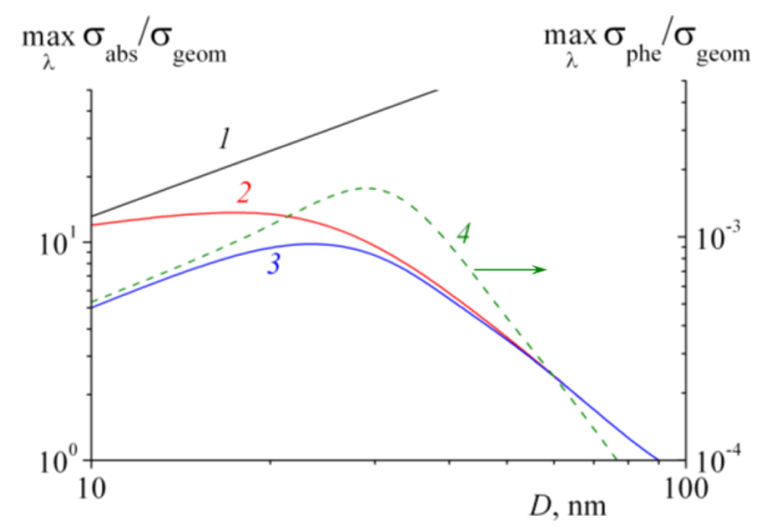

Figure 7. Dependence of the normalized absorption and photoemission cross sections of the sphere on its diameter at the wavelength of the maximum of dipole resonance. 1-Absorption cross section without taking into account both collisions of photoelectrons with the nanoparticle boundary and radiation losses. The following curves take into account radiation losses: 2-absorption cross section without collisions of photoelectrons with the nanoparticle boundary; 3 and 4respectively, absorption and photoemission cross sections with electron collisions taken into consideration.

Figure 7), because radiation correction strongly $\left(\propto n_{\text {matr }}{ }^{3}\right)$ depends on the refractive index of the matrix.' With the radiation correction the quantity $\max _{\lambda}\left(\sigma_{\mathrm{abs}}(\lambda) / \sigma_{\text {geom }}\right)$ takes the maximum at some diameter $D$ located in the region of several tens of nanometers (about $25 \mathrm{~nm}$ for curve 3 in Figure 7). If the collisions with the metal-semiconductor interface are not taken into account, the optimal diameter moves to $20 \mathrm{~nm}$ (the maximum of curve 2). As it is expected, both collision and collisionless models approach each other for big particles, when the electron collisions with the surface have a minor effect on absorption. The optimal diameter for the photoemission is near $30 \mathrm{~nm}$ (curve 4). It is greater than that for the maximum of absorption in conformity with the results of Figure 6: photoemission peak is shifted, but the size parameter of the sphere $\pi D / \lambda$ defining the light scattering has the same value. Faster decrease of the photoemission compared to the absorption in Figure 7 is caused by $\eta_{\mathrm{i}}(\lambda)$. It also makes the electric dipole peak to vanish in the photoemission spectrum for $D>60 \mathrm{~nm}$.

4.2. Truncated Cone. To characterize the shape of truncated cone, we introduce the conicity parameter $\zeta=1-$ $R_{1} / R_{2}$ (see the insert in Figure 8). The height and shape of the plasmon peak depend on $\zeta$. Figure 8 shows the evolution of normalized absorption cross-section spectrum, when the conicity parameter rises from 0 to 0.9 (the cylinder is transformed to the cone). One observes that the absorption peak is decreased and split into two peaks with increasing $\zeta$. Hence, the cylinder $(\zeta=0)$ is the best for absorption. By the analogy with the spherical nanoparticle, we can conclude that the peak at the shorter (longer) frequencies corresponds to the electric quadrupole (dipole) resonance. The red shift of the resonances for bigger $\zeta$ can be understood as follows. At $\zeta=0$, the whole volume $V_{\mathrm{c}}$ of the cylindrical nanoparticle is filled with metal possessing permittivity $\varepsilon_{\mathrm{i}}=1-\omega_{\mathrm{p}}^{2} / \omega^{2}$, where $\omega_{\mathrm{p}}$ is the plasma frequency. For $\zeta>0, V_{\mathrm{c}}$ is filled also with the dielectric of matrix $\varepsilon_{\mathrm{e}}$. Then, the effective permittivity of cylindrical particle can be estimated as $\varepsilon_{\text {eff }}=f \varepsilon_{\mathrm{e}}+(1-f) \varepsilon_{\mathrm{j}}$, where $0 \leq f \leq$ 1 is the fill fraction of the dielectric in the volume of cylinder. Using the condition of plasmonic resonance in cylinders $\varepsilon_{\text {eff }}=$

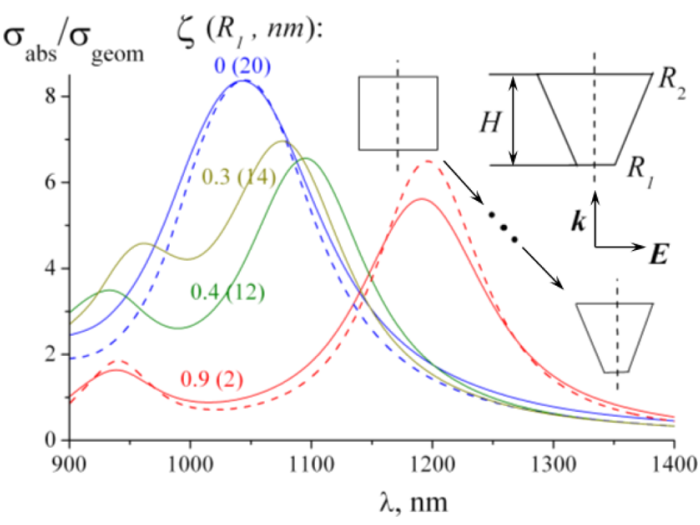

Figure 8. Evolution of the spectrum of normalized absorption cross section with increase of conicity parameter $\zeta$ of truncated cone (or with decrease of smaller base radius $R_{1}$ ) indicated near the curves. Dashed curves for $\zeta=0.9$ and $\zeta=0$ are calculated without the correction for collisions of photoelectrons with the nanoparticle boundary. $R_{2}=20 \mathrm{~nm} ; H=40 \mathrm{~nm}$.

-1 , we can estimate the resonant wavelength $\lambda_{\mathrm{r}}=\left(2 \pi c / \omega_{\mathrm{p}}\right)^{2}[1$ $\left.+\left(1+f \varepsilon_{\mathrm{e}}\right) /(1-f)\right]$. The less metal (the larger conicity parameter), the higher resonant wavelength.

As well as for spheres, the collisions of photoelectrons with the surface of the nanoparticle lead to the lower and broader plasmonic resonances.

Figure 9 demonstrates that plasmon peaks in the absorption $\sigma_{\text {abs }}$ and photoemission $\sigma_{\text {phe }}$ cross sections are quite close to

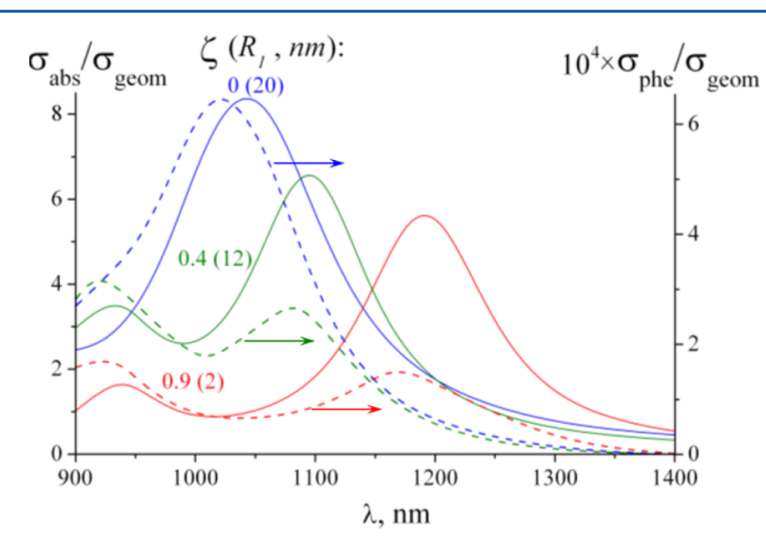

Figure 9. Spectra of the normalized absorption and photoemission cross sections for various shapes of truncated cone nanoparticles determined by the conicity parameter $\zeta$ (smaller base radius) indicated near the curves. Absorption and photoemission cross sections are shown, respectively, by solid and dashed curves of the same color for the same $\zeta$. $R_{2}=20 \mathrm{~nm} ; H=40 \mathrm{~nm}$.

each other at smaller wavelengths and deviate from each other for greater $\zeta$ and longer wavelengths. The quadrupole (i.e., the shorter wavelength) peak in the photoemission spectrum in Figure 9 for cones $(\zeta>0)$ is stronger and, therefore, more advantageous than the dipole one.

4.3. Cylinder. Here, we consider cylindrical nanoparticles with equal diameter and height $D_{\text {cyl }}=H_{\text {cyl }}$ and investigate the effect of the linear nanoparticle size on the photoemission efficiency (the cylinder corresponds to parameters $R_{1}=R_{2}=$ $D_{\text {cyl }} / 2$ in the inset of Figure 8 ).

Maximum of the normalized absorption cross section is reached at the cylinder diameter $D_{\text {cyl }}$ near $30 \mathrm{~nm}$. The increase and decrease of $\sigma_{\mathrm{abs}} / \sigma_{\text {geom }}$ are shown in Figure 10a,b, 

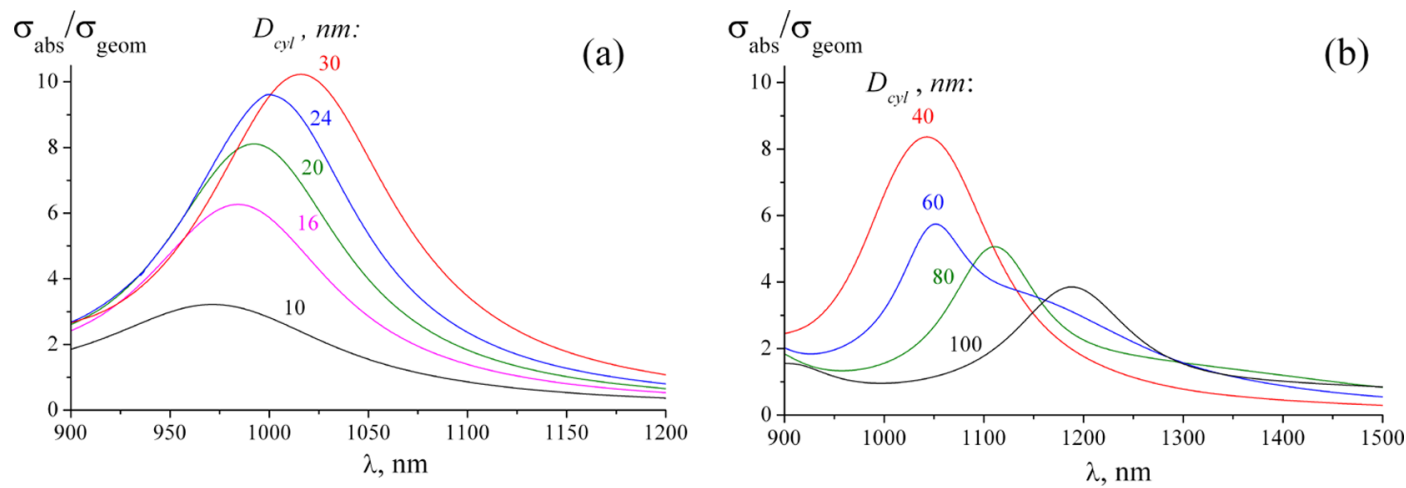

Figure 10. (a) Increase and (b) decrease of the maximum value of the nanocylinder absorption cross section vs its linear dimension $D_{\text {cyl }}=H_{\text {cyl }}$.

respectively. When the cylinder is small, the absorbed energy scales as $D_{\text {cyl }}^{3}$ (the correction for radiation can be neglected in this case), resulting in the linear dependence of the normalized cross section as $\sigma_{\text {abs }} / \sigma_{\text {geom }} \propto D_{\text {cyl }}{ }^{3} / D_{\text {cyl }}{ }^{2}=D_{\text {cyl }}$. In spite of the small dimensions of the metallic nanoparticles, the permittivity of metal turns to be great enough to excite the electric quadrupole oscillations. This makes the electric dipole approximation (Rayleigh approximation) inapplicable. The field inside the nanoparticle becomes inhomogeneous for the bigger cylinders and absorbed energy scales slower than $D_{\text {cyl }}^{2}$, explaining the decrease of $\sigma_{\text {abs }} / \sigma_{\text {geom }}$ with $D_{\text {cyl }}$ shown in Figure $10 \mathrm{~b}$.

As demonstrated in Figure 11, the peak values of the absorption and photoemission cross sections are quite close to

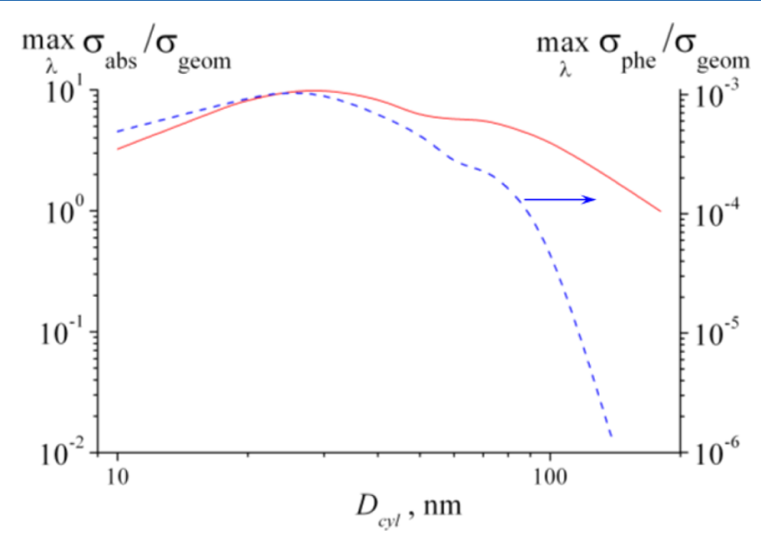

Figure 11. Dependence of the maximum values of normalized absorption and photoemission cross sections on cylinder's diameter $D_{\text {cyl }}\left(D_{\text {cyl }}=H_{\text {cyl }}\right)$.

each other for the small nanocylinders with diameters less than 20-30 nm. This means that the optimal for the photoemission values of the cylinder size can be well-predicted using only the absorption cross section. However, the absorption and photoemission cross sections of bigger cylinders are quite different because of two phenomena. The first one is the decrease of the electron transmission coefficient (see eq 5) through the nanoparticle-matrix interface, when $D_{\text {cyl }}$ increases. The second factor is related to the increase of the electron mean free path for greater $\lambda_{\mathrm{r}}$ and, therefore, larger $D_{\text {cyl }}$. These phenomena all together decrease the probability of photoelectrons to escape the nanoparticle in accordance with eq 4 and, hence, strongly reduce the PCS at larger $D_{\text {cyl }}$ as shown in Figure 11 .

\section{CONCLUSIONS}

We have developed the theoretical model and computational tool for calculation of the bulk PCS into the semiconductor matrix. It has allowed us to optimize the PCS for spheroidal, cylindrical, and truncated-cone gold nanoparticles. The model takes into account several features that cannot be neglected for adequate computation of the bulk photoemission, namely the jump of the photoelectron effective mass at the nanoparticlematrix interface, cooling of the hot photoelectrons due to electron-electron collisions, and modification of the dielectric constant of the nanoparticle metal owing to the collisions of photoelectrons with the nanoparticle boundary. These three effects explain magnitude of the photoemission and optimized values of the nanoparticle geometrical parameters.

Dependencies of the IQE of photoemission on the aspect ratio of semiaxes of spheroidal nanoparticles have been calculated for different values of the model parameters (jump of the effective mass and cooling length). It has been shown that the maximum of the IQE for the spheroidal shape is reached in the case of the sphere. Maximum of the PCS of the sphere is achieved at the dipole localized plasmon resonance peak for the diameter around $30 \mathrm{~nm}$. For the bigger nanoparticles, the quadrupole resonances dominate, while the dipolar photoemission peak vanishes, being shifted to the longer wavelengths. We have revealed that the maximum of the PCS for a truncated cone nanoparticle corresponds to the cylinder. The optimum size of the cylinder is $D_{\text {cyl }}=H_{\text {cyl }} \approx 25$ $\mathrm{nm}$.

Electron-electron scattering processes explain the peculiarities in dependencies of the absorption and photoemission cross sections on the size of the nanoparticle. In particular, a correlation between the spectrum of absorption and photoemission cross sections has been shown for all three shapes of nanoparticles considered. This correlation deteriorates as the plasmon peak approaches the red boundary of the photoemission owing to decrease of the photoemission probability. The plasmon resonance peaks of the absorption and photoemission cross sections are close to each other. This paves new ways for optimization of the PCS by means of the optimization of absorption cross section, what is less computationally demanding.

As a future work, a comparison between the surface and volume (bulk) photoemission from nanoparticles of various shapes can be carried out using the similar theoretical and numerical procedures. 


\section{AUTHOR INFORMATION}

\section{Corresponding Author}

*E-mail: rihsanov@hse.ru. Phone: +7 (906) 728-88-42.

\section{ORCID $\odot$}

Renat Sh. Ikhsanov: 0000-0003-0207-2959

Alexander V. Uskov: 0000-0001-8816-4103

Notes

The authors declare no competing financial interest.

\section{ACKNOWLEDGMENTS}

The work of R.S.I. was financially supported by joint RussianGreek projects RFMEFI61717X0001 and T4 $\Delta$ P $\Omega-00031$ "Experimental and theoretical studies of physical properties of low-dimensional quantum nanoelectronic systems". Also, the publication was prepared within the framework of the Academic Fund Program at the National Research University Higher School of Economics (HSE) in 2018-2019 (grant no 18-05-0024) and by the Russian Academic Excellence Project "5-100". A.V.N. acknowledges partial financial support from the Villum Fonden (DarkSILD project) and the Belarusian Republican Foundation for Fundamental Research (grant no. F16R-049). A.V.U. acknowledges financial support from Russian Science Foundation (Grant 17-19-01532). The work of I.E.P. was done with support from Russian Foundation for Basic Research (Grant 17-58-150007).

\section{REFERENCES}

(1) Brongersma, M. L.; Halas, N. J.; Nordlander, P. Plasmon-induced Hot Carrier Science and Technology. Nat. Nanotechnol. 2015, 10, 2534.

(2) Govorov, A. O.; Zhang, H.; Demir, H. V.; Gun'ko, Y. K. Photogeneration of Hot Plasmonic Electrons with Metal Nanocrystals: Quantum Description and Potential Applications. Nano Today 2014, 9, 85-101.

(3) Uskov, A. V.; Protsenko, I. E.; Ikhsanov, R. S.; Babicheva, V. E.; Zhukovsky, S. V.; Lavrinenko, A. V.; O’Reilly, E. P.; Xu, H. Internal Photoemission from Plasmonic Nanoparticles: Comparison Between Surface and Volume Photoelectric Effects. Nanoscale 2014, 6, 47164727.

(4) Ikhsanov, R. S.; Babicheva, V. E.; Protsenko, I. E.; Uskov, A. V.; Guzhva, M. E. Bulk Photoemission from Metal Films and Nanoparticles. Quantum Electron. 2015, 45, 50-58.

(5) Sousa-Castillo, A.; Comesaña-Hermo, M.; Rodríguez-González, B.; Pérez-Lorenzo, M.; Wang, Z.; Kong, X.-T.; Govorov, A. O.; Correa-Duarte, M. A. Boosting Hot Electron-driven Photocatalysis through Anisotropic Plasmonic Nanoparticles with Hot Spots in Au$\mathrm{TiO}_{2}$ Nanoarchitectures. J. Phys. Chem. C 2016, 120, 11690-11699.

(6) Maier, S. A. Plasmonics: Fundamentals and Applications; Springer Science+Business Media LLC, 2007.

(7) Kreibig, U.; Vollmer, M. Optical Properties of Metal Clusters; Springer Series in Materials Science 25; Springer: Berlin, 1995.

(8) Uskov, A. V.; Protsenko, I. E.; Mortensen, N. A.; O’Reilly, E. P. Broadening of Plasmonic Resonance Due to Electron Collisions with Nanoparticle Boundary: a Quantum Mechanical Consideration. Plasmonics 2014, 9, 185-192.

(9) Protsenko, I. E.; Uskov, A. V. Photoemission from Metal Nanoparticles. Phys.-Usp. 2012, 55, 508-518.

(10) Chen, Q. Y.; Bates, C. W. Geometrical Factors in Enhanced Photoyield from Small Metal Particles. Phys. Rev. Lett. 1986, 57, 27372740.

(11) Tamm, I.; Schubin, S. Zur Theorie des Photoeffektes an Metallen. Z. Phys. 1931, 68, 97-113.

(12) Tan, S.; Dai, Y.; Zhang, S.; Liu, L.; Zhao, J.; Petek, H. Coherent Electron Transfer at the $\mathrm{Ag} / \mathrm{Graphite}$ Heterojunction Interface. Phys. Rev. Lett. 2018, 120, 126801.
(13) Wu, K.; Chen, J.; McBride, J. R.; Lian, T. Efficient Hot-Electron Transfer by a Plasmon-Induced Interfacial Charge-Transfer Tansition. Science 2015, 349, 632-635.

(14) Stuart, R.; Wooten, F.; Spicer, W. E. Monte Carlo Calculations Pertaining to the Transport of Hot Electrons in Metals. Phys. Rev. 1964, 135, A495-A505.

(15) Schmidt, M.; Brauer, M.; Hoffmann, V. Internal Photoemission in Heterostructures Affected by Scattering Processes. J. Phys. D: Appl. Phys. 1997, 30, 1442-1445.

(16) Frese, K. W., Jr.; Chen, C. Theoretical Models of Hot Carrier Effects at Metal-Semiconductor Electrodes. J. Electrochem. Soc. 1992, $139,3234-3243$

(17) Leenheer, A. J.; Narang, P.; Lewis, N. S.; Atwater, H. A. Solar Energy Conversion via Hot Electron Internal Photoemission in Metallic Nanostructures: Efficiency Estimates. J. Appl. Phys. 2014, 115, 134301.

(18) Zhang, H.; Govorov, A. O. Optical generation of hot plasmonic carriers in metal nanocrystals: the effects of shape and field enhancement. J. Phys. Chem. C 2014, 118, 7606-7614.

(19) Brodsky, A. M.; Gurevich, Y. Y. Theory of External Photoeffect from the Surface of a Metal. Sov. Phys. JETP 1968, 27, 114-121.

(20) Ikhsanov, R. S.; Protsenko, I. E.; Uskov, A. V.; Guzhva, M. E. Dependence of Electron Photoemission from Metallic Nanoparticles upon their Size. J. Russ. Laser Res. 2014, 35, 501-508.

(21) Koropov, A. V. Charge Effects in the Metal-Semiconductor Composite System. Phys. Solid State 2004, 46, 1502-1507.

(22) Sze, S. M. Physics of Semiconductor Devices; Wiley: New York, 1981.

(23) Chen, I.-S.; Jackson, T. N.; Wronski, C. R. Characterization of Semiconductor Heterojunctions Using Internal Photoemission. J. Appl. Phys. 1996, 79, 8470-8474.

(24) Myrtveit, T. Modelling of Photoresponse in Metal-Semiconductor Junctions. Appl. Surf. Sci. 1993, 73, 225-231.

(25) Hohenester, U.; Trügler, A. MNPBEM - A Matlab Toolbox for the Simulation of Plasmonic Nanoparticles. Comput. Phys. Commun. 2012, 183, 370-381.

(26) Johnson, P. B.; Christy, R. W. Optical Constants of the Noble Metals. Phys. Rev. B: Solid State 1972, 6, 4370-4379. 